\title{
A 19-Year-Old Man with Dyspnea and Stridor after Surgery
}

\author{
Kim Vanderheyde Thierry Pieters Daniel Rodenstein \\ Service de pneumologie, Cliniques Universitaires Saint-Luc, Université Catholique de Louvain, Bruxelles, Belgique
}

\section{Case Report}

A 19-year-old man with Crouzon's disease was admitted to our hospital for the neurosurgical correction of a cervico-occipital hinge, cure for Arnold-Chiari type 2 malformation and lumbar drain positioning.

The immediate result was excellent and the patient was extubated without complications the next morning. His neurological status as estimated by the Glasgow Coma Scale was 15/15 just after extubation and the lumbar drain was under aspiration at $5 \mathrm{ml} / \mathrm{h}$.

On the seventh day after surgery, he developed acute dyspnea and stridor without hyperthermia.

\section{Medical History}

The patient's medical history was dominated by Crouzon's disease. Crouzon's disease is a progressive polymalformative disease, transmitted in an autosomal dominant pattern (mutation of the FGFR2 gene in $60 \%$ of cases), associated with craniostenosis and hypoplasia of the facial bones. In 1993, the patient was operated for craniostenosis and benefited from a ventriculoperitoneal drain. He regularly presented bronchial infections.

Fig. 1. Chest X-ray performed on the seventh and the eleventh day after surgery showing partial atelectasis of the left lung.

\section{Physical Examination and Additional Examinations}

The patient's physical examination revealed stridor with diffuse polyphonic crackles on auscultation, normal blood pressure, a rapid and steady heart rate (148 bpm), and low oxyhemoglobin saturation at $90 \%$ on the pulse oximeter despite 2 liters per minute of additional oxygen and a $37.5^{\circ} \mathrm{C}$ temperature.

Chest X-ray showed, besides a significant known sinistro-convex scoliosis, partial atelectasis of the left lung (fig. 1). Blood analysis showed only a minimal inflammatory pattern (CRP level $1.2 \mathrm{mg} / \mathrm{dl}$ and hyperleucocytosis $15,800 / \mu l$, neutrophilic), and arterial blood gases disclosed hypoxemia without hypercapnia despite supplemental oxygen ( $\mathrm{pH} 7.44, \mathrm{pCO}_{2} 43 \mathrm{~mm} \mathrm{Hg}, \mathrm{pO}_{2} 50 \mathrm{~mm}$ $\mathrm{Hg}$, and oxyhemoglobin $85.1 \%$ ).

What is your diagnosis?
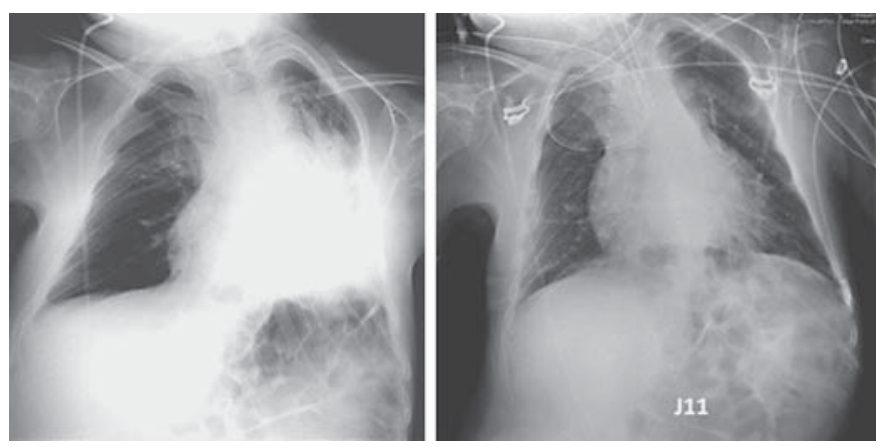

Thierry Pieters, MD

Service de pneumologie

Cliniques Universitaires Saint-Luc, UCL

BE-1200 Brussels (Belgium)

Tel. +32 2764 2832, Fax +32 2764 2831, E-Mail Thierry.Pieters@ uclouvain.be 
2

Fig. 2. OFTP seen through FVB.

Fig. 3. Extraction of OFTP with a tripod clamp.

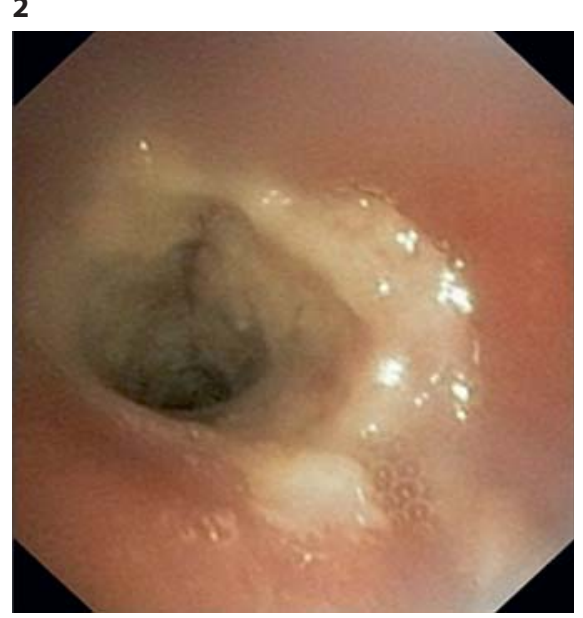

3

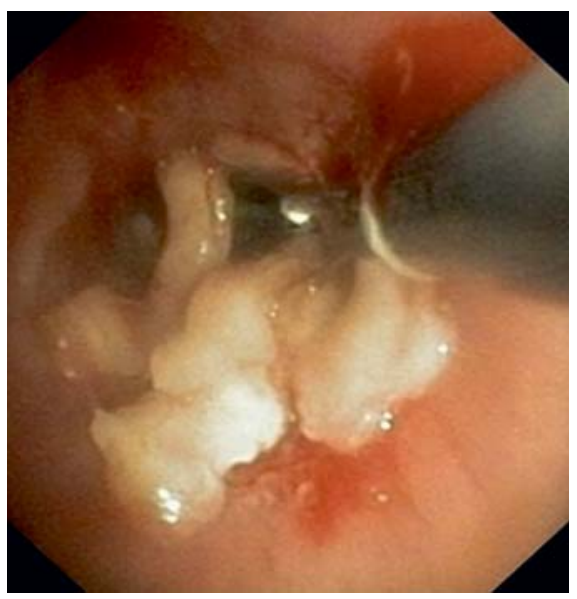

\section{Diagnosis: Obstructive Fibrinous Tracheal Pseudomembranes}

\section{Clinical Course}

As coughing and physiotherapy were ineffective, a diagnostic flexible video-bronchoscopy (FVB) was proposed in order to obtain bacteriological samples and aspirate any possible mucous plug. This was accepted by the patient and his family on day 8 . Informed consent was obtained from the patient. The endoscopy was performed under local anesthesia without sedation. The FVB showed a completely redesigned anatomy of the oral cavity, normal vocal cords, and a distorted trachea with whitish pseudomembranes over the entire length of the trachea. The examination was complicated by a significant desaturation of up to $79 \%$ despite supplementation with 10 liters of oxygen per minute.

An interventional FVB under general anesthesia was performed the following day with laryngeal mask intubation. Vertebral instability due to a recent surgical cure for Arnold-Chiari malformation strictly contraindicated rigid bronchoscopy. We discovered a very posterior epiglottis in the supine position, making the passage through the vocal cords very difficult, as well as a laryngeal distortion with the trachea in an S shape. Beneath the vocal cords, circumferential pseudomembranes covered the entire lumen (fig. 2, 3). We performed a near total removal of the pseudomembranes using usual biopsy forceps, i.e. bipod, tripod, balloon, and basket forceps. The procedure lasted about $2 \mathrm{~h}$. The time course was marked by ventilation difficulties related to the breakdown edema of the upper airway and vocal cords requiring instant intubation until day 23.
Bronchial washing reported the presence of rare oxacillin-sensitive Staphylococcus aureus . Pathological analysis showed large fibrin-leukocyte clusters without evidence of fungal colonization and, on the surface of the fibrin-leukocyte membranes, small foci of local bacterial colonization. The patient left the hospital on day 41 .

\section{Discussion}

Obstructive fibrinous tracheal pseudomembranes (OFTP) are considered a rare but potentially fatal complication of endotracheal intubation. To our knowledge this condition had been described only a few times [1-3], but this case report is the first to describe lesions that extend the entire length of the trachea and are not only located in the area of contact between the cuff and the mucosa.

OFTP were first reported by Harbison et al. [1] in 1999 and were first described by Deslee et al. [2] who reported 10 cases in 2000. In their series, the average duration of endotracheal intubation was 6.2 days, but could be less than $24 \mathrm{~h}$. The first symptoms appeared $3 \mathrm{~h}$ to 9 days after extubation. A continuous or intermittent stridor was the main symptom. There was 1 death.

Various injuries related to endotracheal cuffs have been described [4], mostly attributed to the duration of intubation and to the pressure transmitted to the tracheal wall [5]. Other contributing factors are also listed in table 1 . 
Table 1. Risk factors for injuries related to the tracheal cuff during invasive mechanical ventilation

Risk factors for tracheal lesions

Cuff contact overpressure

Duration of intubation

Use of inert material

Size and caliber of the endotracheal tube

Movements of the endotracheal tube

Nasogastric tube feeding

Hypotension during anesthesia or ventilation

Local infection

Steroid use

Tracheal geometry anatomy

Sequela lesion from previous surgery or radiotherapy

Cervical trauma

Gastric fluid reflux

Unusual reaction to inert material

The FVB showed thick, tubular, rubber-like, whitish pseudomembranes molding the tracheal wall and firmly adhering to it. All these pseudomembranes could be easily detached en bloc from the tracheal wall by the distal tip of a rigid bronchoscope and removed with forceps. Pathology showed fibrinous material with polymorphonuclear infiltration and, in several cases, patchy areas of desquamated necrotic tracheal epithelium. Evolution to a normal tracheal mucosa without stenotic lesions could be achieved.

The main accepted hypothesis for OFTP is that they could represent the first stage of an ischemic process which ultimately could result in tracheal stenosis. Overpressure in the cuff at the site of contact of the endotracheal tube compared with the perfusion pressure of the tracheal mucosa will induce lesions of ischemic necrosis in the mucosa and submucosa leading to the exposure of cartilage, chondritis, and perichondritis.

Our patient developed OFTP after a short-duration endotracheal intubation. The absence of hyperthermia, biological inflammation, immunosuppression, drugs, and clinical and bacteriological contributions may reasonably exclude a fungal or bacterial infection. Several other pathophysiological mechanisms could explain the fact that the injuries were located along the entire length of the trachea and not only in the area of contact between the cuff and the mucosa and emerged in waning following extubation. Firstly, from an anatomical point of view, the congenital malformation of the facial bones may have contributed in a certain way to a difficult intubation and, therefore, to potential damage to the entire tracheal mucosa. Secondly, the tracheal deformation in an S shape could have contributed to friction between the endotracheal cuff and the tracheal wall during its insertion and could have led to an underestimation of the pressure of the endotracheal cuff. Thirdly, this could have torn the tracheal wall during flexion-extension of the neck in surgery and during the transfer of the patient to the intensive care unit; quickly increasing cuff pressure induces ischemic lesions. Fourthly, the particular vessel distribution, i.e. the arterial supply and vascularization of the thoracic trachea, is mainly supplied by the thoracic branch of the inferior thyroid artery (or Haller's thoracic branch). Whether the main bronchi are supplied by another vascularization directly issued from the thoracic aorta could explain ischemic injuries involving only the thoracic trachea and sparing the mucosa under the carina, like in our case.

All of these physico-anatomical constraints, favored by the absence of monitoring pressure inside the cuff tube and the use of a small volume-high pressure cuffed tube (Blue Line tube; Portex Limited, Kent, UK), may have induced tracheal mucosa vascular hypoperfusion secondary to an intense inflammatory reaction similar to the skin lesions of pressure necrosis due to hyperpressure and friction at the points of pressure.

OFTP have also been described in several other conditions. Infection is the most prevalent etiology both in children and adults (croup, tracheobronchitis, staphylococcal infection in children, and diphtheria) [6-8]. The majority of the cases were reported in immunocompromised patients as a complication of invasive pulmonary aspergillosis or rare cases of nondiphtheria corynebacteria [9-11]. Other clinical situations can also lead to the formation of pseudomembranes, such as malignant tumors.

The diagnosis of OFTP is made by FVB as blood sampling and imaging are inconclusive. Positive evolution after the removal of the OFTP without recurrence and the absence of antibiotics or antifungals are highly supportive of the hypothesis of a causal relationship with shortduration intubation rather than a postinfectious etiology.

In conclusion, in any patient presenting stridor following extubation, even after a short period of intubation, OFTP should be suspected given its rapid and potentially fatal evolution. Differentiation from glottic edema, acute bronchial obstruction, or laryngospasm is important because of the specific treatment for OFTP versus corticosteroids. 
The diagnostic procedure of choice is bronchoscopy, which is intended to both diagnose and cure. The removal of OFTP is always curative without long-term sequelae.

\section{Conclusions}

OFTP are a very rare cause of potentially fatal stridor following extubation, even after a short-duration (less than $24 \mathrm{~h}$ ) endotracheal intubation.

OFTP were first described in 1999 and the main hypothesis is that they could represent the first stage of an ischemic process related to excessive cuff pressure.

The investigation procedure of choice is bronchoscopy, which is intended to both diagnose and cure. The removal of OFTP is always curative without long-term sequelae.
The pressure of the tracheal cuff should ideally average $25 \mathrm{~cm} \mathrm{H}_{2} \mathrm{O}$ and should not exceed $30 \mathrm{~cm} \mathrm{H}_{2} \mathrm{O}$. The state of the art is the use of a high volume-low pressure cuff with continuous monitoring pressure inside the cuff endotracheal tube [12].

\section{Key Words}

Stridor - Pseudomembrane - Flexible video bronchoscopy

\section{Financial Disclosure and Conflicts of Interest}

The authors report no personal or financial conflict of interest.

\section{References}

1 Harbison J, Collins D, Lynch V, McNicholas WT: Acute stridor due to upper tracheal membrane following endotracheal intubation. Eur Respir J 1999;14:1238.

2 Deslee G, Brichet A, Lebuffe G, et al: Obstructive fibrinous tracheal pseudomembrane: a potentially fatal complication of tracheal intubation. Am J Respir Crit Care Med 2000;162:1169-1171.

$\checkmark 3$ Casoni GL, Coffa A, Gurioli C, et al: A severe complication of short-term tracheal intubation. Anesth Analg 2007;104:232-233.

$\checkmark 4$ Nordin U: The trachea and cuff-induced tracheal injury: an experimental study on causative factors and prevention. Acta Otolaryngol Suppl 1977;345:1-71.
5 Seegobin RD, van Hasselt GL: Endotracheal cuff pressure and tracheal mucosal blood flow: endoscopic study of effects of four large volume cuffs. Br Med J (Clin Res Ed) 1984 288:965-968.

6 Henry RL, Mellis CM, Benjamin B: Pseudomembranous croup. Arch Dis Child 1983;58: 180-183.

7 Aaron L, Heurtebise F, Bachelier MN, Guimard Y: Pseudomembranous diphtheria caused by Corynebacterium ulcerans (in French). Rev Med Interne 2006;27:333-335.

-8 Yamazaki Y, Hirai K, Honda T: Pseudomembranous tracheobronchitis caused by methicillin-resistant Staphylococcus aureus. Scand J Infect Dis 2002;34:211-213.

-9 Franco J, Muñoz C, Vila B, Marín J: Pseudomembranous invasive tracheobronchial aspergillosis. Thorax 2004;59:452.
10 Pervez NK, Kleinerman J, Kattan M, Freed JA, Harris MB, Rosen MJ, Schwartz IS: Pseudomembranous necrotizing bronchial aspergillosis: a variant of invasive aspergillosis in a patient with hemophilia and acquired immune deficiency syndrome. Am Rev Respir Dis 1985;131:961-963.

-11 Tomita M, Hasegawa M, Akutsu Y, Okudaira T: Pseudomembranous bronchitis (nondiphtherial) resulting in sudden death: an autopsy report. Intern Med 1992;31:933-935.

12 Nseir S, Duguet A, Copin MC, De Jonckheere J, Zhang M, Similowski T, Marquette $\mathrm{CH}$ : Continuous control of endotracheal cuff pressure and tracheal wall damage: a randomized controlled animal study. Crit Care 2007;11:R109. 